\title{
SHIPBORNE WIND MEASUREMENT AND MOTION-INDUCED ERROR CORRECTION BY COHERENT DOPPLER LIDAR OVER YELLOW SEA IN 2014 Xiaochun Zhai ${ }^{1}$, Songhua $\mathrm{Wu}^{1,2 *}$, Bingyi Liu ${ }^{1}$, Xiaoquan Song ${ }^{1}$ \\ ${ }^{1}$ Ocean Remote Sensing Institute, Ocean University of China, China, *wush@ouc.edu.cn ${ }^{2}$ Laboratory for Regional Oceanography and Numerical Modeling, Qingdao National Laboratory for Marine Science and Technology, China
}

\begin{abstract}
Shipborne wind observations by the Coherent Doppler Lidar (CDL) during the 2014 Yellow Sea campaign are presented to study the structure of the Marine Atmospheric Boundary Layer (MABL). This paper gives an analysis of the correction for horizontal and vertical wind measurement, demonstrating that the combination of the CDL with the attitude correction system enables the retrieval of wind profiles in the MABL during both anchored and cruising measurement with satisfied statistical uncertainties.
\end{abstract}

\section{INTRODUCTION}

The vertical structure of atmospheric variables in Marine Atmospheric Boundary Layer (MABL) is significant for understanding the driving and coupling mechanisms and for parameterizing ocean-atmosphere interaction process. The Coherent Doppler Lidar (CDL) is an available tool with high spatial and temporal resolution, providing nearly continuous particle backscatter, wind profile observations in clear atmosphere [1], which is demanding for the air-sea boundary layer turbulent characteristics. Unlike the conventional wind measuring methods, CDL can only detect the Line-Of-Sight (LOS) velocity which is the projection of the horizontal and vertical velocity to the Lidar beam direction, thus it is necessary to conduct measurement at three or more different directions of the probing beam to retrieve the wind velocity vector. More complicated attitude correction should be considered when CDL is carried out at moving platform such as the ship or aircraft since the orientation of transmitting laser beam is not fixed and the speed of the ship itself and ocean wave will be stacked to the LOS velocity which has more obvious effect on vertical velocity. There have been several studies on attitude correction and error analysis of velocity measured by CDL. Wolfe et al and Pichugina et al [2] deployed the NOAA High Resolution Doppler Lidar (HSRL) along with the first use of a motion compensation system at sea in 2004. Hill et al [3] used the NOAA HSRL with a Strapped-Down System (SDS) to compensate for the orientation of the Lidar's scanning unit for the ship's motion and concluded that the attitude correction depends on the velocity of the seatainer and on the motion of the hemispheric scanner relative to the seatainer. Wulfmeyer et al corrected the vertical velocity using LOS velocity in zenith stare mode and horizontal wind derived from Velocity Azimuth Display (VAD) mode.

In order to measure the wind field and turbulence characteristics of MABL of the Yellow Sea, the experimental investigation was undertaken by Dongfanghong-2 research vessel affiliated with Ocean University of China in 2014. This paper gives a brief analysis of the correction for horizontal and vertical wind measurement using CDL with attitude correction system and robust motion correction algorithm.

\section{METHODOLOGY}

Ship motion turns out to be an important error source for the determination of turbulence variables using CDL. To study boundary layer dynamics, the atmospheric velocity in Earth coordinate system is required, so the correction of the Doppler frequency shift for the ship's motion relative to the Earth coordinate system is required, where the direction of the laser beam in Earth coordinate system and the LOS velocity induced by ship movement need to be determined using attitude correction system (ACS). Generally, the ACS uses Global Navigation Satellite System (GNSS) to define Earth coordinate system, and the ship speed, heading angle and earth location including the longitude and latitude in Earth coordinate system can be obtained from GNSS. 
Another important part of ACS is the inertial navigation system, which records the ship motion angles including pitch, roll, laser beam azimuth and elevation in ship coordinate system.

Figure 1 shows the flowchart of data process of shipborne CDL. Specifically, the LOS velocity and Signal to Noise Ratio (SNR) can be firstly determined using Lidar data and FFT analysis. After the data pre-processing including the quality control based on SNR threshold and averaging process, the attitude transformation is then used to obtain the azimuth and elevation in each LOS vector in Earth coordinate system. The LOS velocity detected by Lidar is the atmosphere motion relative to ship coordinate system, thus the removal of the ship motion speed projection on the measured LOS is needed. Finally, the horizontal and vertical wind profiles can be retrieved using modified 4-DBS mode and zenith stare mode data, respectively.

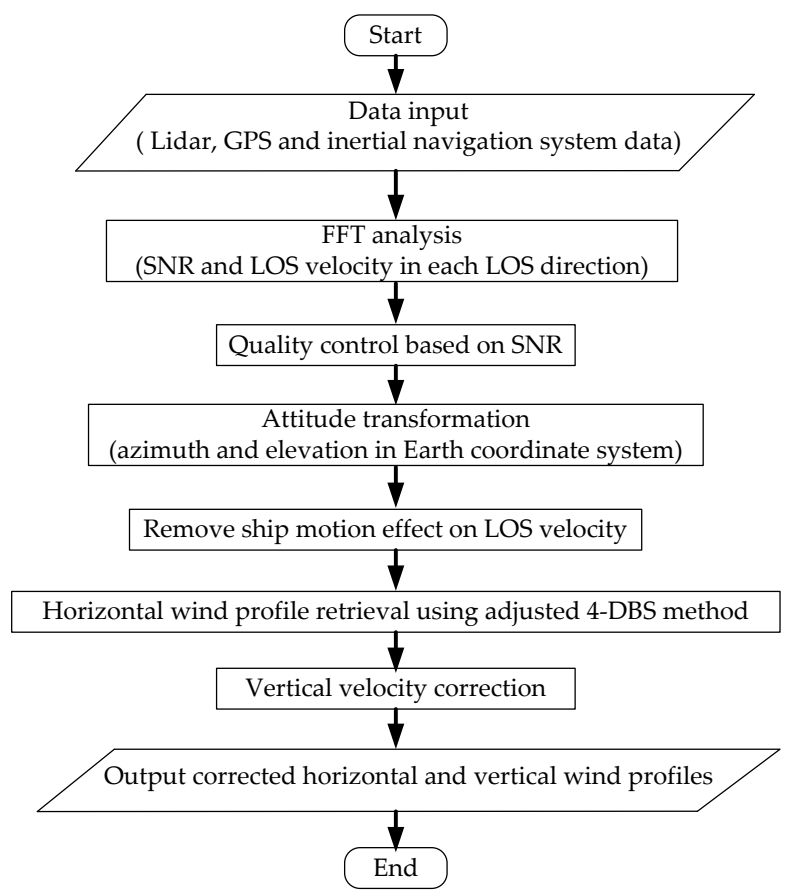

Figure 1 Flow chart of ship motion correction algorithm based on $C D L$

\section{RESULTS}

\subsection{Horizontal wind evaluation}

Two examples of obtained horizontal wind profiles are plotted in Figure 2 for anchored measurements and in Figure 3 for cruising observations, respectively. Meanwhile, the Lidar data (blue curves) are also compared to the radiosonde data (red curves), which were averaged over at least $10 \mathrm{~min}$ after the launch of the radiosonde. In Figure 2, The Root-MeanSquare-Error ( RMSE) in speed between Lidar and radiosonde below $2 \mathrm{~km}$ are $0.49 \mathrm{~ms}^{-1}$ for the uncorrected measurement and $0.45 \mathrm{~ms}^{-1}$ for the corrected measurements, both showing consistent with the radiosonde wind speed, which is reasonable since the effect of ship speed on LOS velocity is less in anchored measurement. However, the RMSE in wind direction between Lidar and radiosonde are $84.43^{\circ}$ for uncorrected measurement and $5.27^{\circ}$ for corrected measurement. The obvious difference of the uncorrected measurement in wind direction is mainly derived from two aspects. The first one results from the definition in different coordinate systems, where the heading has an important effect on Lidar azimuth. The second aspect is that because of the experimental field limitation, the direction of GNSS master antenna is perpendicular to the ship bow, meaning that the "real" heading is the recorded heading plus $90^{\circ}$. Generally, the attitude correction is necessary especially for the wind direction retrieval when the ship is anchored with slight shake.
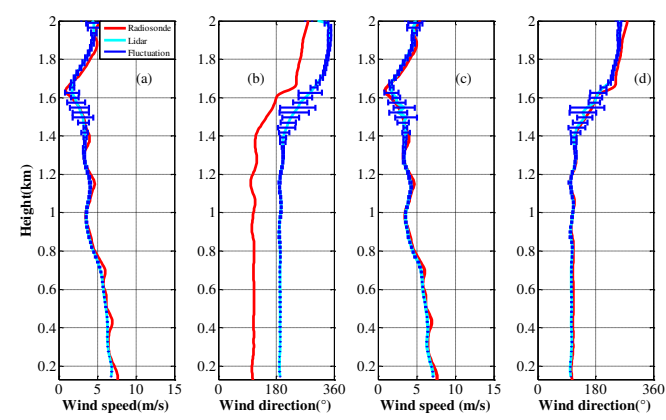

Figure 2 Anchored observation: (a) (c) wind speed and (b) (d) wind direction measured by CDL (blue line) before and after attitude correction, respectively. The simultaneous radiosonde data is shown in red line. The blue bars represent the sampling fluctuations from 15:52 to 16:02 LT (Local Time), 09 May, 2014.

Figure 3 shows the results of the cruising observation from 07:44 to 07:54 LT on 13 May 2014. In this case, the ship roll has more effect on the Lidar elevation when it points to port or 
starboard, on the contrary, the Lidar elevation in bow or stern direction is more sensitive to ship pitch. The RMSE in speed between Lidar and radiosonde data below $1.0 \mathrm{~km}$ are $4.42 \mathrm{~ms}^{-1}$ for uncorrected measurement and $0.88 \mathrm{~ms}^{-1}$ for corrected measurements, and the corresponding $R M S E$ in wind direction are $48.71^{\circ}$ and $9.52^{\circ}$, respectively. Therefore, the attitude correction algorithm has obviously improved the wind profile results when the ship is in cruising measurement. It is noted that the discrepancies in wind speed and direction above $1.0 \mathrm{~km}$ between radiosonde and corrected CDL may result from multipath effects at the ship's platform.
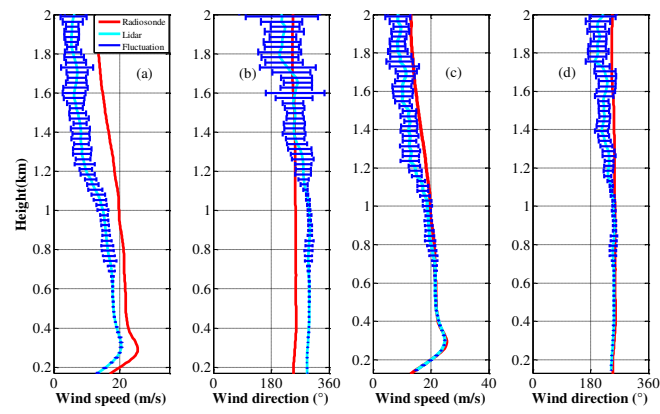

Figure 3 As figure 2, but for 07:44 to 07:54 LT 13 May, 2014 in cruising observation.

In order to assess the accuracy of the Lidar wind retrievals, results have been compared to the 11 radiosonde dataset during the experiment. Figure 4 shows scatter plots of wind speed and direction for Lidar and radiosonde using modified 4-DBS solution. Both wind speed and direction show negligible bias, demonstrating the feasibility and reliability of the modified 4-DBS method.
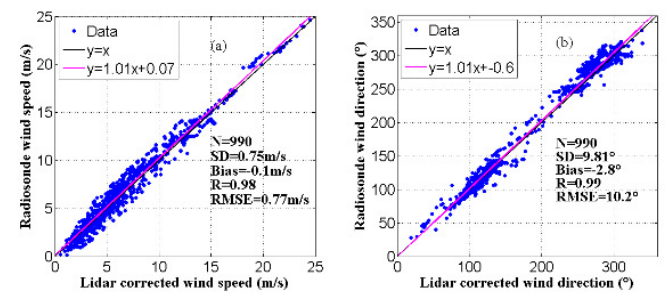

Figure 4 Comparison of $(a)$ wind speed and $(b)$ wind direction between CDL and radiosonde data from 09 May 2014 to 19 May 2014.

\subsection{Vertical wind evaluation}

The motion correction of vertical velocity is more obvious compared with the horizontal wind components. A typical measurement of 14 May 2014 is presented in Figure 5. Figure 5 (a) shows the whole series of time-height cross sections of the SNR. The MABL height is also presented in black solid circles. Figure 5 (b) shows the time series of ship heading, CDL laser beam azimuth and elevation, and horizontal wind direction at $H=0.4 \mathrm{~km}$. During the zenith stare mode, the mean angle between ship heading and the laser azimuth is $66^{\circ}$ with standard deviation of $7^{\circ}$, thus the projection of ship velocity on vertical velocity is always positive, the results of which are shown in Figure 6 (a). Furthermore, the estimation of the horizontal wind speed and direction (black line in Figure 5 (b)) from modified 4-DBS solution is used to substract the horizontal wind speed projection $\overrightarrow{r_{g}} \cdot \vec{V}$ from the relative speed measured by CDL. In this case, the $\overrightarrow{r_{g}} \cdot \vec{V}$ is positive and negative in downwind and headwind, respectively, causing the overestimate and underestimate of the vertical velocity, the effect of which is shown in Figure 6 (b). The resulting vertical velocity wind speed are presented in Figure 5 (c). The red and blue color indicates positive (upward) and negative (downward) movement, respectively, of the atmosphere parcels along the laser beam. It has been noted that the vertical velocity has a significant diurnal variation. Specifically, the downdrafts dominants mixing layer in the morning and amounted to about $0.5 \mathrm{~ms}^{-1}$, and small-scale convective activity can be observed at the top of mixing layer. As the solar radiation strengthens, the atmospheric convection becomes more active and extends to the whole mixing layer, the strengths of updrafts and downdrafts are weakly stronger than before and the atmospheric vertical alternation becomes more frequent. The mixing layer recovers to descending motions with a continuous and long period after 13:11 LT. The difference between the corrected and uncorrected vertical velocity is shown in Figure. 6 (c), obviously showing the temporal and spatial variation of the contributions of ship motion and horizontal wind on vertical velocity. 

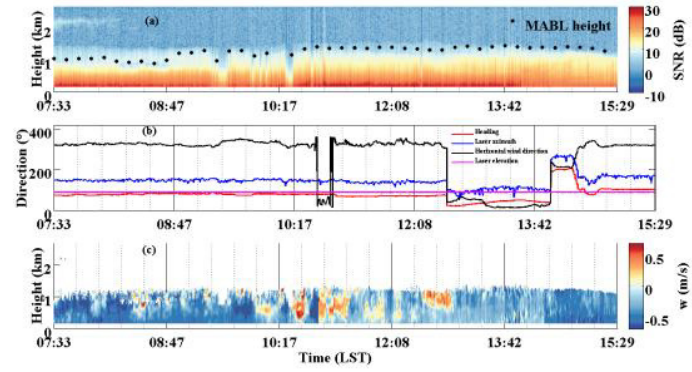

Figure 5 Example measurement from 07:33 to 15:29 14 May 2014: (a) Time-Height-Intensity of SNR and retrieved MABL height using SNR gradient method (black solid circles) (b) time series of ship heading, CDL laser beam azimuth and elevation in the Earth coordinate system, and horizontal wind direction at 0.4 km. (c) Time-Height-Intensity of vertical velocity after attitude correction.

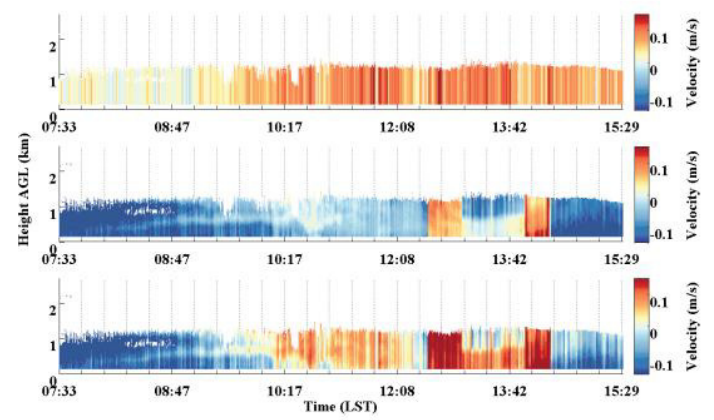

Figure 6 Vertical velocity correction analysis: (a) projection of ship velocity on vertical velocity $(b)$ the effect of horizontal wind on vertical velocity (c) difference between vertical velocity after attitude correction and vertical velocity before attitude

\section{CONCLUSIONS}

Shipborne wind observation by the CDL during the 2014 Yellow Sea campaign has been presented to study the structure of the MABL. The attitude correction system of CDL consists of GNSS and inertial navigation system to directly measure the speed and the attitude of the ship. According to the transformation matrix from the product of roll, pitch and heading rotation matrix, the azimuth and elevation of the LOS velocity in the Earth coordinate system can be firstly determined. Then the removal of the ship speed projection on the measured LOS velocity is needed to obtain the "real" LOS velocity in Earth coordinate system. The horizontal wind profiles can be retrieved by a modified 4-DBS of CDL velocity data. For the case of vertical velocity, small deviations from vertical pointing due to ship motion and pointing angle knowledge errors introduces a projection of the horizontal wind on the LOS vector, thus estimations of the horizontal wind speed contribution are used to correct the vertical velocity.

The comparison of the CDL to radiosonde shows that attitude correction is essential for the wind retrieval in cruising measurement. The correlation coefficients of wind speed and direction are 0.98, 0.99 , respectively, both of which shows negligible bias and demonstrates the feasibility and reliability of the modified 4-DBS method. A case study of 8-h time series observation on 14 May 2014 is presented to compare uncorrected and corrected vertical velocity, additionally showing the specific temporal and spatial variation of the contributions of ship motion and horizontal wind on vertical velocity. Generally, the combination of CDL with attitude correction system and accurate motion correction process has the potential of continuous long-term high-spatial and temporal resolution measurement for MABL thermodynamic and turbulence process.

\section{ACKNOWLEDGEMENTS}

This work was supported by the National Natural Science Foundation of China (NSFC) under grant 41471309 and 41375016 and the National High Technology Research and Development Program of China (No. 2014AA09A511).

\section{References}

[1] Wu, S. H., Liu, B. Y., Liu, J. T., Zhai, X. C., Feng, C. Z., Wang, G. N., Zhang, H. W., Yin, J. P., Wang, X. T., Li, R. Z., Gallacher, D., 2016: Wind turbine wake visualization and characteristics analysis by Doppler lidar, Opt. Express, 24 (10), A762-A780.

[2] Wolfe, D., Brewer, W., Tucker, S., White, A., White, D., Welsh, D., Ruffieux, D., Fairall, C., Ratterree, M., and Intrieri, J., 2007: Shipboard multisensor merged wind profiles from the New England Air Quality Study 2004, J. Geophys. Res.: Atmos., 112.

[3] Hill, R. J., Brewer, W. A., and Tucker, S. C., 2008: Platform-motion correction of velocity measured by Doppler lidar, J. Atmos. Oceanic. Technol., 25, 1369-1382. 